\title{
Efecto de la adición de antioxidantes sobre la motilidad espermática post-criopreservación y fertilidad del semen de peces
}

\author{
Rodríguez, M.; Nivia, A. \\ Escuela de Ciencias Agrícolas, Pecuarias y del Medio Ambiente (ECAPMA), \\ Universidad Nacional Abierta y a Distancia (UNAD), Sede Nacional \\ Calle 14 Sur N ${ }^{\circ}$ 14-23, Bogotá, CP 110911, Colombia, PBX: \\ (+57)1344 3700. E-mail: alexander.nivia@unad.edu.co
}

\begin{abstract}
Resumen
Rodríguez, M.; Nivia, A.: Efecto de la adición de antioxidantes sobre la motilidad espermática post-criopreservación y fertilidad del semen de peces. Rev. vet. 28: 2, 157-164, 2017. La criopreservación de semen produce daños celulares a nivel de membranas plasmáticas, mitocondria y ADN, debido principalmente a la formación de cristales de hielo intra y extracelulares, así como al estrés osmótico y oxidativo generado. Para minimizar estos daños se utilizan medios diluyentes que simulan las características fisiológicas del semen y contienen sustancias crioprotectoras, las cuales con ayuda de algunos antioxidantes proporcionan una mayor supervivencia espermática post-descongelación y mejoría de las tasas de motilidad y fertilidad. La adición de antioxidantes enzimáticos como superóxido dismutasa, catalasa y peróxidasa durante el proceso de criopreservación de células espermáticas en peces, no favorece las tasas de motilidad espermática post-descongelación ni de fertilización en las especies trucha arco iris y de arroyo, mientras que los no enzimáticos (methylenediphosphonic-acid/MDPA, butil-hidroxitolueno/BHT, cisteína, propóleo, ácido ascórbico, lisina y carnitina) las mejoran de forma significativa en especies como esturión beluga, carpa común, esturión ruso y trucha arco iris. Los antioxidantes producen diversos efectos especie-específicos tanto en variables de calidad espermática como fertilidad y su efectividad depende de la concentración y el medio diluyente utilizado. Se concluye que la utilización de antioxidantes no enzimáticos en la criopreservación de semen de especies acuícolas como carpa común, esturión y trucha, resulta indispensable para mejorar la motilidad y fertilidad espermática.
\end{abstract}

Palabras clave: peces, antioxidantes, criopreservación, motilidad, fertilidad.

\begin{abstract}
Rodríguez, M.; Nivia, A.: Effect of the addition of antioxidants on the spermatic motility post-cryopreservation and fertility of the semen of fish. Rev. vet. 28: 2, 157-164, 2017. Semen cryopreservation produces cellular damage to the plasma membranes, mitochondria and DNA mainly due to the formation of intra- and extracellular ice crystals, as well as due to the osmotic and oxidative stress generated. To minimize this damage, diluent mediums are used. They simulate the physiological characteristics of semen and contain cryoprotectants and some antioxidants which provide increased post-thaw sperm survival and improve motility and fertility rates. The addition of enzymatic antioxidants such as superoxide dismutase, catalase and peroxidase during cryopreservation of sperm cells in fish do not improve rates sperm motility post-thawed neither fertilization in the species rainbow trout and brook trout, whereas non-enzymatic (methylenediphosphonic-acid/MDPA, butil-hidroxitolueno/BHT, cysteine, propolis, ascorbic acid, lysine and carnitine) significantly improved such parameters in species such as beluga sturgeon, common carp, Russian sturgeon and rainbow trout. Antioxidants produce different effects on species-specific variables sperm quality and fertility and their effectiveness depends on the concentration and the diluent medium used. It can be concluded that the use of non-enzymatic antioxidants in semen cryopreservation aquaculture species such as common carp, sturgeon and trout are essential to improve sperm motility and fertility.
\end{abstract}

Key words: fish, antioxidants, cryopreservation, motility, fertility. 


\section{Introducción}

La criopreservación del semen de peces es una técnica de gran interés para la piscicultura, ya que permite la conservación de gametos tanto de especies productivas como de aquéllas en vía de extinción, así como crear bancos de germoplasma ${ }^{16}$.

Sin embargo, este proceso produce daños celulares en los espermatozoides, tanto a nivel de la membrana plasmática como en las mitocondrias y el ácido desoxiribonucleico (ADN), debido a la formación de cristales de hielo intra y extracelularmente, así como también por el estrés osmótico y oxidativo que provocan los procesos de congelación y descongelación ${ }^{73}$.

Para minimizar estos daños se han utilizado diversos medios diluyentes que no solo permiten su almacenamiento por largos periodos de tiempo, sino que también simulan las características fisiológicas del esper$\mathrm{ma}^{55}$. Estos medios diluyentes contienen a su vez una o más sustancias crioprotectoras como dimetilsulfóxi$\mathrm{do}^{22}$, metanol ${ }^{39}$, dimetilacetamida ${ }^{45}$ y metilenglicol ${ }^{36}$.

\section{Los antioxidantes}

Con el fin de proporcionar una mayor sobrevivencia espermática post-descongelación, se han utilizado diversos tipos de antioxidantes de índole enzimática y no enzimática, los cuales favorecen la respuesta de variables como la motilidad, viabilidad e integridad del $\mathrm{ADN}^{46}$.

Adicionalmente, se ha reportado que combaten el estrés oxidativo generado por el desbalance entre la producción y eliminación de las especies reactivas de oxigeno (ERO) durante los procesos de congelación y descongelación ${ }^{62}$.

También se ha encontrado que el uso de antioxidantes como el butil hidroxitolueno (BHT) en los medios de criopreservación de semen de algunas especies, mejoran los porcentajes de fertilidad, expresando una mejor tasa de producción de embriones y por ende de eclosión.

\section{Objetivo}

La presente revisión tiene como objetivo comparar las propiedades y respuestas de los diferentes tipos de antioxidantes que han sido utilizados en protocolos de crioconservación de semen de las especies acuícolas: trucha arco iris (Oncorhynchus mykiss), trucha de arroyo (Salvelinus fontinalis), esturión ruso (Acipenser gueldenstaedtii), esturión beluga (Huso huso) y carpa común (Ciprynus carpio), así como el efecto que producen sobre la fertilidad y la motilidad espermática post-criopreservación.

\section{Funciones de los antioxidantes}

Un antioxidante se define como una sustancia que cuando está presente en bajas concentraciones, en com- paración con un sustrato oxidable, reduce o inhibe significativamente la oxidación de dicho sustrato ${ }^{31}$.

Los antioxidantes tienen diversas funciones, como no permitir que moléculas se unan al oxígeno, al reaccionar e interactuar más rápido con las especies reactivas de oxígeno en un determinado microambiente ${ }^{31}$, dismutar el oxígeno para formar el peróxido de hidrógeno y proteger a las células contra el anión superóxido, y regular la permeabilidad de la membrana plasmática impidiendo su peroxidación lipídica ${ }^{72}$.

\section{Usos en criopreservación}

Los antioxidantes se usan como aditivos de los medios diluyentes durante el proceso de criopreservación, para aumentar el éxito del protocolo y obtener mejores tasas de motilidad y fertilidad espermática $9,18,58$.

En células espermáticas son usados para mitigar los efectos de la criopreservación y adicionalmente retardar o prevenir la oxidación de la membrana lipídica causada por radicales libres, los cuales se producen en la mitocondria a través de la nicotinamida adenina dinucleótido (NAD) dependiente de la vía oxidoreductasa y en la misma membrana a través de nicotinamida adenina di-nucleótido fosfatasa (NADP+) dependiente del sistema oxidasa, durante el proceso de congelación y descongelación ${ }^{1,3}$.

\section{Efectos sobre las células espermáticas}

Como producto del metabolismo celular se producen grandes cantidades de radicales libres ${ }^{7}$, los cuales se encuentran clasificados como EROs (anión superóxido, anión peróxido, radical perhidroxilo, radical hidroxilo) y como especies reactivas de nitrógeno (óxido nítrico, radical peroxinitrito).

Estas sustancias son producidas por mitocondrias, lisosomas, peroxisomas, membrana nuclear, citoplasma y retículo endoplásmico; sin embargo, también son generadas por factores externos como la contaminación ambiental, la exposición a radiaciones ionizantes, el tabaco, los medicamentos, los aditivos químicos en alimentos procesados y algunos pesticidas, herbicidas $y$ fungicidas ${ }^{60}$.

Cuando se realiza la criopreservación de células espermáticas, el sistema antioxidante enzimático (superóxido dismutasa, catalasa y glutatión peroxidasa) se activa convirtiendo al anión superóxido $\left(\mathrm{O}_{2}-\right)$ en peróxido de hidrógeno $\left(\mathrm{H}_{2} \mathrm{O}_{2}\right)$ e impidiendo la aparición de iones hidroxilos, altamente tóxicos ${ }^{2}$. El peróxido de hidrógeno atraviesa los compartimientos celulares sin interactuar con el NADPH, ácidos nucleicos, proteínas ni lípidos, siendo usado por las células para su maduración y capacitación ${ }^{68}$.

Por su parte, el sistema de defensa no enzimática está compuesto por una serie de diversos compuestos químicos que son incorporados a través de la dieta, los cuales cumplen algunas funciones como impedir las reacciones en cadena producidas por el radical hidro- 
peróxido durante la peroxidación lipídica ${ }^{48}$ o proteger a las lipoproteínas de la membrana plasmática contra la oxidación ${ }^{69}$.

\section{Uso en criopreservación de espermatozoides de peces}

Diversos estudios en peces han evaluado el efecto de la adición de antioxidantes a los medios diluyentes, sobre el porcentaje de motilidad espermática post-descongelación y su fertilidad, los cuales se han llevado a cabo en especies como: trucha arco iris ${ }^{38,40}$, trucha de arroyo ${ }^{40}$, esturión ruso ${ }^{44}$, esturión beluga ${ }^{52} \mathrm{y}$ carpa común ${ }^{49,50,51}$. Los antioxidantes evaluados fueron de dos tipos: enzimáticos y no enzimáticos.

\section{Antioxidantes enzimáticos y no enzimáticos}

Los antioxidantes enzimáticos catalasa, superóxido dismutasa y peroxidasa se han evaluado en los salmónidos trucha arco iris ${ }^{38,40} \mathrm{y}$ trucha de arroyo ${ }^{40}$. Los antioxidantes no enzimáticos tipo vitamina (C, E y trolox) se han utilizado en las especies trucha arco iris ${ }^{38}$, esturión ruso ${ }^{44} \mathrm{y}$ esturión beluga ${ }^{52}$; los tipos aminoácido (lisina, metionina, cisteína y taurina) en trucha arco iris ${ }^{22,38,40}$, esturión ruso ${ }^{44}$, y carpa común ${ }^{49}$.

Los compuestos naturales $\beta$-caroteno y propóleo se han valorado en trucha arco iris ${ }^{38}$ y carpa común ${ }^{49}$, los compuestos sintéticos (3,5-di-ter-butil-4-hidroxifenil (MDPA) y butilhidroxitolueno (BHT) en esturión beluga y carpa común ${ }^{50,52}$ y las sustancias químicas ácido úrico, carnitina y glutatión en sus formas reducida y oxidada, fueron ensayadas en las especies trucha arco iris ${ }^{38,40} \mathrm{y}$ de arroyo ${ }^{40}$.

\section{Utilidad en la criopreservación de semen}

La adición de los diferentes tipos de antioxidantes a los medios diluyentes ha producido diversos efectos especie-específicos en parámetros como la motilidad y fertilidad de los espermatozoides de peces.

Anteriores estudios han reportado que los antioxidantes no enzimáticos (vitamina $\mathrm{C}$, lisina, cisteína, metionina, vitamina E, glutatión reducido, L-carnitina, ácido úrico, propóleo, MDPA, BHT, mezcla glutatión reducido y oxidado) lograron optimizar la función mitocondrial en los procesos de criopreservación de esperma de cuatro especies de peces (Tabla 1).

Sin embargo, los antioxidantes como vitamina $\mathrm{C}$, lisina, cisteína, propóleo, MDPA, BHT, mezcla glutatión reducido y oxidado y L-carnitina, aumentaron de forma simultánea las tasas de motilidad espermática post-descongelación y de fertilización (Tabla 2).

Otros estudios mostraron un efecto diverso al utilizar las vitaminas las $\mathrm{C}$ y $\mathrm{E}$ como antioxidantes en los procesos de criopreservación de espermatozoides de especies animales como conejos ${ }^{74}$, bovinos ${ }^{26,32}$, equinos ${ }^{27}$, porcinos y humanos ${ }^{14,67}$. Resultados similares fueron obtenidos al utilizar como antioxidantes
Tabla 1. Antioxidantes no enzimáticos que optimizaron la función mitocondrial durante el proceso de criopreservación del semen de peces.

\begin{tabular}{llcc}
\hline especie & antioxidante & MC & MA \\
\hline esturión ruso & vitamina C, 0,01 mM & 32 & 38 \\
\hline trucha arco iris & metionina, $1,5 \mathrm{mM} / \mathrm{L}$ & 32 & 65 \\
\hline esturión ruso & lisina, 0,05 mM & 32 & 36 \\
\hline carpa común & cisteína, 2,5 mM & 46,67 & 50 \\
& cisteína, 5 mM & 46,67 & 64,67 \\
& cisteína, $20 \mathrm{mM}$ & 46,67 & 76 \\
\hline trucha arco iris & GSH, 1,5 mmol/1 & 32 & 42 \\
& GSH+GSSG, 3,0 mM & 21,2 & 22,9 \\
& L-carnitina,0,05 mM/1 & 32 & 57 \\
& ácido úrico, 0,25 mM/1 & 32 & 62 \\
\hline carpa común & propóleo, 0,2 mg/ml & 40,3 & 54,3 \\
& propóleo, 0,4 mg/ml & 40,3 & 55 \\
& propóleo, 0,6 mg/ml & 40,3 & 61,7 \\
& propóleo, 0,8 mg/ml & 40,3 & 68,7 \\
& propóleo, $1 \mathrm{mg} / \mathrm{ml}$ & 40,3 & 63,3 \\
\hline esturión beluga & MDPA, 0,1 mM & 20 & 50 \\
& BHT, 0,1 mM & 20 & 40 \\
\hline carpa común & BHT, 0,0001 mM & 45,7 & 54,3 \\
& BHT, 0,001 mM & 45,7 & 72,9 \\
& BHT, 0,01 mM & 45,7 & 81,4 \\
& BHT, 0,1 mM & 45,7 & 51,4 \\
\hline trucha arco iris & vitamina E, 2 mmol/1 & 32 & 53 \\
\hline & & & \\
& &
\end{tabular}

GSSG: glutatión oxidado; GSH: glutatión reducido; MDPA: 3,5-di-ter-butil-4-hidroxifenil; BHT: butilhidroxitolueno, MC: tasa de motilidad espermática posdescongelación del control; MA: tasa de motilidad espermática post-descongelación con el antioxidante. Elaborada en base a datos de Mirzoyan $2006{ }^{44}$, Kutluyer $2014{ }^{38}$, Öğretmen $2014{ }^{49}$, Öğretmen \& Inanan $2014{ }^{50}$, Osipova $2014{ }^{52}$, Öğretmen $2015^{51}$.

los aminoácidos lisina, cisteína y metionina, en células espermáticas de caprinos ${ }^{70,71}$ y bovinos ${ }^{13}$.

De igual forma, se reportó el mismo efecto cuando se utilizó propóleo y BHT en espermatozoides de caprinos ${ }^{18,34,47}$ y porcinos ${ }^{56}$, así como l-carnitina en seres humanos ${ }^{8}$, felinos ${ }^{41}$, caprinos ${ }^{71}$ y roedores ${ }^{53}$, el glutatión en su forma reducida (GSH) en personas ${ }^{29} \mathrm{y}$ porcinos ${ }^{28}$, el glutatión oxidado (GSSH) y la mezcla de glutatión reducido y oxidado en bovinos ${ }^{13}$.

El efecto benéfico de antioxidantes como cisteína, lisina, metionina, ácido ascórbico y carnitina, representado por el aumento de la motilidad, podría estar relacionado con el hecho de que la cisteína -al ser adicionada a medios diluyentes- refuerza el aumento de la producción de glutatión, tanto intra como extracelular, lo cual ayuda a prevenir la pérdida de la motilidad espermática debida a la formación de iones peróxido ${ }^{13}$.

Asímismo, la lisina junto con metionina y ácido ascórbico, son esenciales para la biosíntesis de carnitina, la cual es un antioxidante fundamental para metabolizar los ácidos grasos de cadena larga en el interior del citosol de las mitocondrias, donde al ser degradados se convierten en energía (ATP) para ser luego utilizada 
Tabla 2. Antioxidantes no enzimáticos que aumentaron de forma simultánea las tasas de motilidad espermática post-descongelación y de fertilización en peces.

\begin{tabular}{llcccc}
\hline especie & antioxidante & MC & MA & FC & FA \\
\hline esturión ruso & vitamina C, 0,01mM & 32 & 38 & 55 & 78 \\
& lisina, 0,05mM & 32 & 36 & 55 & 63 \\
\hline carpa común & cisteína, 2,5mM & 46,67 & 50 & 96 & 96 \\
& cisteína, 5mM & 46,67 & 64,67 & 96 & 96,33 \\
& cisteína, 20mM & 46,67 & 76 & 96 & 97 \\
\hline trucha arco iris & GSH+GSSG, 3,0mM & 21,2 & 22,9 & 87 & 92 \\
& L-carnitina,0,05mM/1 & 32 & 57 & 86,71 & 90,01 \\
\hline carpa común & propóleo, 0,2mg/ml & 40,3 & 54,3 & 84,7 & 92,7 \\
& propóleo, 0,4 mg/ml & 40,3 & 55 & 84,7 & 92,7 \\
& propóleo, 0,6 mg/ml & 40,3 & 61,7 & 84,7 & 94,3 \\
& propóleo, 0,8mg/ml & 40,3 & 68,7 & 84,7 & 94,7 \\
& propóleo, 1mg/ml & 40,3 & 63,3 & 84,7 & 95 \\
\hline esturión beluga & MDPA, 0,1mM & 20 & 50 & 30 & 60 \\
& BHT, 0,1mM & 20 & 40 & 30 & 40 \\
\hline carpa común & BHT, 0,0001mM & 45,7 & 54,3 & 69,3 & 75 \\
& BHT, 0,001mM & 45,7 & 72,9 & 69,3 & 76 \\
& BHT, 0,01mM & 45,7 & 81,4 & 69,3 & 79 \\
& BHT, 0,1 mM & 45,7 & 51,4 & 69,3 & 87,3 \\
\hline
\end{tabular}

GSSG: glutatión oxidado; GSH: glutatión reducido; MDPA: 3,5-di-terbutil-4-hidroxifenil; BHT: butilhidroxitolueno; MC: tasa de motilidad espermática posdescongelación del control; MA: tasa de motilidad espermática post-descongelación con el antioxidante; FC: tasa de fertilidad del control; FA: tasa de fertilidad con el antioxidante. Elaborada en base a datos de Mirzoyan $2006^{44}$, Kutluyer $2014{ }^{38}$, Öğretmen $2014^{49}$, Öğretmen \& Inanan $2014^{50}$, Osipova $2014{ }^{52}$, Öğretmen $2015^{51}$.

por las células espermáticas para aumentar su motilidad ${ }^{15}$. Adicionalmente, la carnitina ayuda a proteger la membrana fosfolipídica del espermatozoide contra la peroxidación lipídica ${ }^{53}$.

De igual forma, el efecto reportado por el propóleo podría estar relacionado con la inducción de la activación de enzimas como la superóxido dismutasa ${ }^{33}$ y la catalasa ${ }^{66}$, las cuales protegen al organismo del ataque de radicales y al ADN contra el daño oxidativo producido por el malondialdehído durante la peroxidación lipídica ${ }^{59}$.

De otra parte, MDPA, BHT y alfa tocoferol, dado que son compuestos fenólicos estéricamente impedidos, funcionan como antioxidantes atrapando los radicales oxi y peroxi ${ }^{35}$. Las altas tasas de motilidad y fertilidad reportadas con el uso de MDPA y BHT se deben a la propiedad redox que tienen estos compuestos permitiendo la absorción y neutralización de los radicales libres, protegiendo a las células espermáticas de la oxidación y degradación prematura y brindándoles una estabilidad frente los cambios bruscos de temperatura ${ }^{52}$. Otros autores le atribuyen excelentes resultados al efecto sincrónico de antioxidantes fenólicos estéricamente impedidos con el medio diluyente yema de huevo ${ }^{30,54}$.
También se ha descubierto que los antioxidantes como la metionina reducida, la mezcla de metionina reducida y oxidada y el glutatión oxidado, produjeron menores tasas de motilidad espermática post-descongelación, sin afectar de forma negativa las tasas de fertilización (Tabla 3).

En estos casos se puede inferir que tales antioxidantes produjeron una fragmentación del ADN, la cual provocó la disminución de la motilidad espermática sin afectar las tasas de fertilización, debido a que en algunas especies de peces y mamíferos, los ovocitos pudieron haber activado el sistema enzimático de reparación del ADN propio actuando sobre el ADN de los espermatozoides, reparándolo y permitiendo que se realice la fertilización y el normal desarrollo de los embriones ${ }^{5,37}$.

La utilización del glutatión, tanto individual como combinada en sus formas reducida y oxidada, produjo resultados variables debido a su concentración durante el proceso de criopreservación (Tabla 4).

Se ha reportado que durante la criopreservación los niveles de glutatión decrecen en los espermatozoides ${ }^{28,29}$. Siendo así, al añadir altas cantidades para compensar el efecto se evidenciaría una alteración de la homeostasis celular. Un investigador reportó que el glutatión se encuentra dentro de las células en una alta concentración $(5-10 \mathrm{mM})$, predominando la forma reducida (GSH) sobre la oxidada (GSSG) y que cuando se produce un aumento o disminución en la proporción de alguno de ellos, es afectado el equilibrio interno de la célula ${ }^{21}$.

Por el contrario, se ha descubierto que los antioxidantes enzimáticos como catalasa, superóxido dismutasa y peróxidasa, así como los no enzimáticos (trolox, taurina y $\beta$-caroteno) no lograron mejorar de forma significativa las tasas de motilidad y fertilidad en peces.

Tabla 3. Antioxidantes no enzimáticos que produjeron menores tasas de motilidad espermática post-descongelación sin afectar de forma negativa las tasas de fertilización.

\begin{tabular}{llcccc}
\hline especie & antioxidante & MC & MA & FC & FA \\
\hline trucha de arroyo & RMet, 1,5mM & 22,2 & 19,7 & 67,9 & 75,2 \\
& RMet+ OxMet, 1,5mM & 22,2 & 21,3 & 67,9 & 71,1 \\
\hline trucha arco iris & RMet+ OxMet, 1,5mM & 21,2 & 21,7 & 87 & 88,7 \\
& RMet+ OxMet,3,0mM & 21,2 & 17,2 & 87 & 85,4 \\
& GSSG,1,5mM/L & 32 & 30 & 86,71 & 88,16 \\
\hline
\end{tabular}

GSSG: glutatión oxidado; OxMet: metionina oxidada; RMet: metionina reducida; MC: tasa de motilidad espermática post-descongelación del control; MA: tasa de motilidad espermática post-descongelación con el antioxidante; FC: tasa de fertilidad del control; FA: tasa de fertilidad con el antioxidante. Elaborada en base a datos de Lahnsteiner $2011^{40}$, Kutluyer $2014{ }^{38}$. 
Tabla 4. Antioxidantes no enzimáticos que presentaron resultados variables durante el proceso de criopreservación.

\begin{tabular}{llcccc}
\hline especie & antioxidante & MC & MA & FC & FA \\
\hline trucha arco iris & GSH, 1,5mmol/1 & 22,2 & 21,3 & 67,9 & 76,3 \\
& GSH, 1,5mmol/1 & 32 & 42 & 86,71 & 87,26 \\
& GSSH,1,5mmol/1 & 32 & 30 & 86,71 & 88,16 \\
& GSH+GSSG, 1,5mM & 21,2 & 17 & 87 & 88,7 \\
& GSH+GSSG, 3,0mM & 21,2 & 22,9 & 87 & 92 \\
\hline trucha de arroyo & GSH+GSSG, 1,5mM & 22,2 & 18 & 67,9 & 57,2 \\
\hline
\end{tabular}

GSSG: glutatión oxidado; GSH: glutatión reducido; MC: tasa de motilidad espermática post-descongelación del control; MA: tasa de motilidad espermática post-descongelación con el antioxidante; FC: tasa de fertilidad del control; FA: tasa de fertilidad con el antioxidante. Elaborada en base a datos de Lahnsteiner $2011^{40}$, Kutluyer $2014^{38}$.

Tabla 5. Antioxidantes enzimáticos y no enzimáticos que no mejoraron de forma significativa las tasas de motilidad y fertilidad en peces durante el proceso de criopreservación.

\begin{tabular}{llcccc}
\hline especie & antioxidante & MC & MA & FC & FA \\
\hline trucha arco iris & SOD 250U/1 & 32 & 60 & 86,71 & 89,23 \\
trucha arco iris & SOD 250U/1 & 21,2 & 16,8 & 87 & 77,1 \\
trucha de arroyo & SOD 250U/1 & 22,2 & 18,4 & 67,9 & 62,1 \\
trucha arco iris & SOD 500U/1 & 21,2 & 10,9 & 87 & 73,4 \\
trucha de arroyo & SOD 500U/1 & 22,2 & 18,3 & 67,9 & 59,8 \\
trucha arco iris & PER 250U/1 & 32 & 22 & 86,71 & 88,61 \\
trucha arco iris & PER 250U/1 & 21,2 & 17,7 & 87 & 90,7 \\
trucha de arroyo & PER, 250U/1 & 22,2 & 17,7 & 67,9 & 68,1 \\
trucha arco iris & PER,500U/1 & 21,2 & 22,6 & 87 & 88,6 \\
trucha de arroyo & PER,500U/1 & 22,2 & 18,7 & 67,9 & 59,8 \\
trucha arco iris & CAT 250U/1 & 32 & 31 & 86,71 & 87,1 \\
trucha arco iris & CAT 250U/1 & 21,2 & 11,1 & $\mathrm{NR}$ & $\mathrm{NR}$ \\
trucha de arroyo & CAT 250U/1 & 22,2 & 13,6 & 67,9 & 43,3 \\
trucha arco iris & CAT 100U/1 & 21,2 & 22,9 & 87 & 89,6 \\
trucha de arroyo & CAT 100U/1 & 22,2 & 22,6 & 67,9 & 75,9 \\
esturión beluga & trolox, 0.1mM & 20 & 10 & 20 & 30 \\
esturión beluga & taurina, 50mm & 61,7 & 63,3 & 69,5 & 63,7 \\
trucha arco iris & taurina, 75mm & 61,7 & 63,3 & 69,5 & 29 \\
trucha arco iris & taurina, 100mm & 61,7 & 3,3 & 69,5 & 9 \\
trucha arco iris & $\beta$-caroteno,0,5mM/L & 32 & 26 & 86,71 & 87,44 \\
\hline
\end{tabular}

SOD: superóxido dismutasa; CAT: catalasa; PER: peroxidasa, MC: tasa de motilidad espermática post-descongelación del control; MA: tasa de motilidad espermática post-descongelación con el antioxidante; FC: tasa de fertilidad del control; FA: tasa de fertilidad con el antioxidante. Elaborada en base a datos de Lahnsteiner $2011^{40}$, Ekici $2012^{22}$, Kutluyer $2014{ }^{38}$, Osipova $2014{ }^{52}$.

Estos resultados han sido similares o inferiores a los obtenidas con los diluyentes estándares (Tabla 5).

Otros estudios mostraron un efecto diverso al utilizar la superóxido dismutasa (SOD) como antioxidante en los procesos de criopreservación de espermatozoides de especies animales como caprinos ${ }^{12,65}$, porcinos ${ }^{57}$, aves ${ }^{4}$, equinos ${ }^{11}$, simios ${ }^{43}$ y caninos ${ }^{19}$. Similares efectos se constataron cuando se empleó la catalasa en espermatozoides humanos ${ }^{46}$, porcinos ${ }^{57}$, bovinos ${ }^{24} \mathrm{y}$ ciervos ${ }^{23}$ $\mathrm{y}$ de igual forma, el trolox en equinos ${ }^{64} \mathrm{y}$ caprinos ${ }^{63}$, la taurina en bovinos ${ }^{20,54} \mathrm{y}$ caprinos ${ }^{6} \mathrm{y}$ finalmente, los carotenoides como la luteína y crocin en semen de porcinos y caprinos 25,42 .

Los antioxidantes enzimáticos no lograron incrementar las tasas de motilidad y fertilidad debido a que las altas concentraciones de las enzimas pudieron ser toxicas para las células. En un estudio se reportó que $50 \mathrm{U} / \mathrm{mL}$ de SOD eran suficientes para mantener la viabilidad espermática postdescongelación en el esperma de gallos; sin embargo, a medida que se acrecentaba la concentración del antioxidante en el medio, aumentaba la toxicidad ${ }^{4}$.

La respuesta contraria presentada por la enzima superóxido dismutasa (250 U/l) en la trucha arco iris, podría haberse debido a la composición del medio diluyente utilizado ${ }^{38,40}$. Un estudio reportó que durante la criopreservación se producen resultados variables utilizando el mismo antioxidante, cuando los medios diluyentes con los que se trabaja poseen distintas composiciones ${ }^{54}$.

Por otra parte, el efecto negativo en cuanto a las tasas de motilidad espermática y fertilidad reportado al utilizar antioxidantes enzimáticos (catalasa, superóxido dismutasa y peróxidasa) y no enzimáticos (trolox, taurina y $\beta$-caroteno), estuvo influenciado por la concentración, dado que no potencializó la función del medio diluyente, ocasionando daños en el ADN mitocondrial y nuclear durante el proceso de la criopreservación, afectando la síntesis de proteínas involucradas en la producción de energía celular y también el proteoma ${ }^{17}$.

En una investigación se reportó la desaparición de 21 proteínas espermáticas después de la criopreservación, las cuales contenían los factores de transcripción de genes importantes no solo para la motilidad sino para la fusión del ovocito con el espermatozoide ${ }^{75}$.

Se afirma que la variabilidad de los resultados obtenidos para los parámetros relacionados con la motilidad espermática y su fertilidad frente a la adición de antioxidantes en los medios diluyentes utilizados en los procesos de criopreservación de espermatozoides, se debe principalmente al tipo de antioxidante y a las diferencias especie-especificas expresadas en el grado de susceptibilidad al estrés oxidativo, así como también al mismo protocolo utilizado durante la criopreservación ${ }^{10,61}$.

\section{Conclusiones}

Los antioxidantes adicionados a los medios diluyentes durante los procesos de criopreservación de esper- 
matozoides presentan propiedades especie-especificas; por lo cual se evidencia una variabilidad en la respuesta de parámetros de calidad espermática y fertilidad.

La respuesta de las variables de motilidad espermática post-criopreservación y fertilidad, se ven afectadas directamente por el tipo de antioxidante utilizado, su concentración y el medio diluyente al que son adicionados.

Los antioxidantes enzimáticos como superóxido dismutasa, catalasa y peroxidasa producen bajas tasas de motilidad espermática post-descongelación, sin mejoría de las tasas de fertilización en las especies acuícolas (truchas arco iris y de arroyo).

Los antioxidantes no enzimáticos como MDPA, BHT, cisteína, propóleo, ácido ascórbico, lisina y carnitina, mejoran de manera significativa la motilidad espermática post-descongelación y las tasas de fertilización en las especies esturión beluga, carpa común, esturión ruso y trucha arco iris.

\section{REFERENCIAS}

1. Aitken RJ, Buckingham D, West K, Wu FC, Zikopoulos K, Richardson DW. 1992. Differential contribution of leucocytes and spermatozoa to the generation of reactive oxygen species in the ejaculates of oligozoospermic patients and fertile donors. $J$ Reprod \& Fert 94: 451-462.

2. Aitken RJ, Roman SD. 2008. Antioxidant systems and oxidative stress in the testes. Oxid Med \& Cel Long 1: 15-24.

3. Agarwal A, Saleh RA, Bedaiwy MA. 2003. Role of reactive oxygen species in the pathophysiology of human reproduction. Fert \& Steril 79: 829-843.

4. Amini MR, Kohram H, Zare A, Zhandi M, Sharideh H, Nabi MM. 2015. The effects of different levels of catalase and superoxide dismutase in modified beltsville extender on rooster post-thawed sperm quality. Cryobiol 70: 226-232.

5. Ashwood MJ, Edwards RG. 1996. Genetics and human conception DNA repair by oocytes. Mol Hum Reprod 2: 46-51.

6. Atessahin A, Bucak MN, Tuncer PB, Kizil M. 2008. Effects of anti-oxidant additives on microscopic and oxidative parameters of Angora goat semen following the freeze-thawing process. Small Rum Res 77: 38-44.

7. Avello M, Suwalsky M. 2006. Radicales libres, antioxidantes naturales y mecanismos de protección. Atenea (Concepción, Chile) 494: 161-172.

8. Banihani S, Agarwal A, Sharma R, Bayachou M. 2014. Cryoprotective effect of 1-carnitine on motility, vitality and DNA oxidation of human spermatozoa. Andrologia 46: 637-641.

9. Baptista CD. 2009. Effect of antioxidants on pot-thaw canine sperm quality. Doctoral Dissertation, Faculdade de Medicina Veterinária e Zootecnia, Universidade de São Paulo. https://bibliotecadigital.ipb.pt/

10. Baumber J, Ball BA, Gravance CG, Medina V, Davies MC. 2000. The effect of reactive oxygen species on equine sperm motility, viability, acrosomal integrity, mitochondrial membrane potential and membrane lipid peroxidation. $J$ Androl 21: 895-902.
11. Baumber J, Ball BA, Linfor JJ. 2005. Assessment of the cryopreservation of equine spermatozoa in the presence of enzyme scavengers and antioxidants. Am J Vet Res 66: 772-779.

12. Berlinguer F, Ledda S, Rosati I, Bogliolo L, Leoni G, Naitana S. 2003. Superoxide dismutase affects the viability of thawed european mouflon semen and the heterologous fertilization using both IVF and intracytoplasmatic sperm injection. Reprod Fertil \& Develop 15: 19-25.

13. Bilodeau JF, Blanchette S, Gagnon C, Sirard MA. 2001. Thiols prevent $\mathrm{H}_{2} \mathrm{O}_{2}$ mediated loss of sperm motility in cryopreserved bull semen. Theriogenology 56: 275-286.

14. Breininger E, Beorlegui NB, O'Flaherty CM, Beconi MT. 2005. Alpha-tocopherol improves biochemical and dynamic parameters in cryopreserved boar semen. Theriogenology 63: 2126-2135.

15. Burke L, Cort M, Cox G, Crawford R, Desbrow B, Farthing L, Minehan M, Shaw N, Warnes O. 2006. Supplements and sports foods, In: Clinical sports nutrition (Burke L, Deakin V, Eds.), 3rd Ed., McGraw-Hill, Chapter 16.

16. Cabrita $\mathbf{E}$ et al. 2010. Cryopreservation of fish sperm: applications and perspectives. $J$ Appl Ichthyol 26: 623-635.

17. Carrasco SC. 2010. Crioconservación: efectos sobre la movilidad espermática y la fertilización en peces. Acta Biol Colomb 15: 2-3.

18. Castilho EF et al. 2009. Use of propolis and ascorbic acid on goat semen cryopreservation. Rev Bras Zoot 38: 2335 2345.

19. Chatdarong K, Chaivechakarn A, Thuwanut P, Ponglowhapan S. 2012. Effects of cold storage prior to freezing on superoxide dismutase, glutathione peroxidase activities, level of total reactive oxygen species and sperm quality in dogs. Reprod Dom Anim 47: 274-277.

20. Chillar S, Singh VK, Kumar R, Atreja SK. 2012. Effects of taurine or trehalose supplementation on functional competence of cryopreserved Karan Fries semen. Anim Reprod Sci 135: 1-7.

21. De Molina MC. 2003. El estrés oxidativo y el destino celular. Química Viva (Buenos Aires), 2: 17-28.

22. Ekici A et al. 2012. Effects of different doses of taurine in the glucose-based extender during cryopreservation of rainbow trout (Oncorhynchus mykiss) semen. Biotech \& Biot Equipm 26: 3113-3115.

23. Fernández MR et al. 2007. Sperm characteristics and DNA integrity of iberian red deer (cervus elaphus hispanicus) epididymal spermatozoa frozen in the presence of enzymatic and nonenzymatic antioxidants. J Androl 28 : 294-305.

24. Fernández M, Domínguez A, Esteso M, Garde J, Martínez F. 2009. Catalase supplementation on thawed bull spermatozoa abolishes the detrimental effect of oxidative stress on motility and DNA integrity. Int J Androl 32: 353 359.

25. Florin VG, Miclea V, Miclea I, Marius Z, Hettig A. 2011. The effect of lutein added in the mangalitza boar cryopreserved semen media. Anim Sci \& Biotech 68: 1-2.

26. Foote RH, Brockett CC, Kaproth MT. 2002. Motility and fertility of bull sperm in whole milk extender containing antioxidants. Animal Reprod Sci 71: 13-23. 
27. Franco JS, Chaveiro A, Góis A, Silva FM. 2013. Effects of $\alpha$-tocopherol and ascorbic acid on equine semen quality after cryopreservation. J Eq Vet Sci 33: 787-793.

28. Gadea J, Sellés E, Marco MA, Coy P, Matás C, Romar R, Ruiz S. 2004. Decrease in glutathione content in boar sperm after cryopreservation: Effect of the addition of reduced glutathione to the freezing and thawing extenders. Theriogenology 62: 690-701.

29. Gadea J, Molla M, Selles E, Marco MA, Garcia FA, Gardon JC. 2011. Reduced glutathione content in human sperm is decreased after cryopreservation: effect of the addition of reduced glutathione to the freezing and thawing extenders. Cryobiology 62: 40-46.

30. Graham JK, Hammerstedt RH. 1992. Differential effects of butylated hydroxytoluene analogs on bull sperm subjected to cold-induced membrane stress. Cryobiology 29: 106-117.

31. Halliwell B. 1995. How to characterize an antioxidant: an update. Biochem Soc Symp 61: 73-101.

32. Hu J, Tian W, Zhao X, Zan L, Wang H, Li Q, Xin Y. 2010. The cryoprotective effects of ascorbic acid supplementation on bovine semen quality. Anim Reprod Sci 121: 72-77.

33. Jasprica I et al. 2007. In vivo study of propolis supplementation effects on antioxidative status and red blood cells. J Ethnopharm 110: 548-554.

34. Khalifa TA, Lymberopoulos AG, Elsaidy BE. 2008. Testing usability of butylated hydroxytoluene in conservation of goat semen. Reprod Dom Anim 43: 525-530.

35. Klein E, Lukes V, Cibulková Z. 2005. On the energetics of phenol antioxidants activity. Petrol \& Coal 47: 33-39.

36. Koch JF, Viveiros AT, Maria AN, Orfao LH. 2007. Diluidores e crioprotetores na criopreservacao do semen de piapara Leporinus obtusidens. Proceed 44th Meet Brazilian Anim Sci Society, Jaboticabal (Brazil), p. 1-3.

37. Kopeika J, Kopeika E, Zhang T, Rawson DM, Holt WV. 2004. Effect of DNA repair inhibitor (3-aminobenzamide) on genetic stability of loach (Misgurnus fossilis) embryos derived from cryopreserved sperm. Theriogenology 61: 1661-1673.

38. Kutluyer F, Kayim M, Öğretmen F, Büyükleblebici S, Tuncer PB. 2014. Cryopreservation of rainbow trout Oncorhynchus mykiss spermatozoa: effects of extender supplemented with different antioxidants on sperm motility, velocity and fertility. Cryobiology 69: 462-466.

39. Lahnsteiner F, Mansour N. 2008. Protocols for the cryopreservation of salmonidae semen. In: Methods in Reproductive Aquaculture: Marine and Freshwater Species Biology Series (Cabrita E, Robles V, Herraez MP Eds.), CRC Press, p. 373-384.

40. Lahnsteiner F, Mansour N, Kunz FA. 2011. The effect of antioxidants on the quality of cryopreserved semen in two salmonid fish, the brook trout (Salvelinus fontinalis) and the rainbow trout (Oncorhynchus mykiss). Theriogenology 76: 882-890.

41. Manee S, Parmornsupornvichit S, Kraiprayoon S, Tharasanit T, Chanapiwat P, Kaeoket K. 2014. L-carnitine supplemented extender improves cryopreserved-thawed cat epididymal sperm motility. Asian-Australasian J Anim Sci 27: 791-796.
42. Mata M, Álvarez M, Tamayo J, Anel L, de Paz P, Martínez F. 2015. Post-thawing quality and incubation resilience of cryopreserved ram spermatozoa are affected by antioxidant supplementation and choice of extender. Theriogenology 83: 520-528.

43. McCarthy MJ, Meyers SA. 2011. Antioxidant treatment in the absence of exogenous lipids and proteins protects rhesus macaque sperm from cryopreservation-induced cell membrane damage. Theriogenology 76: 168-176.

44. Mirzoyan A, Nebesikhina N, Voynova N, Chistyakov V. 2006. Preliminary results on ascorbic acid and lysine suppression of clastogenic effect of deep-frozen sperm of the russian sturgeon (Acipenser gueldenstaedti). Int J Refrig 29: 374-378.

45. Morris JP, Hagen A, Kanki JP. 2008. Zebrafish sperm cryopreservation with N,N-Dimethylacetamide. In: Methods in Reproductive Aquaculture: Marine and Freshwater Species Biology Series (Cabrita E, Robles V, Herraez MP Eds.), CRC Press, p. 329-338.

46. Moubasher AE, Eldin AM, Ali ME, El-sherif WT, Gaber HD. 2013. Catalase improves motility, vitality and DNA integrity of cryopreserved human spermatozoa. Andrologia 45: 135-139.

47. Naijian HR, Kohram H, Shahneh AZ, Sharafi M, Bucak MN. 2013. Effects of different concentrations of BHT on microscopic and oxidative parameters of Mahabadi goat semen following the freeze-thaw process. Cryobiology 66: 151-155.

48. Niki E, Saito T, Kawakami A, Kamiya Y. 1984. Inhibition of oxidation of methyl linoleate in solution by vitamin E and vitamin C. J Biol Chem 259: 4177-4182.

49. Öğretmen F, Inanan BE, Öztürk M. 2014. Protective effects of propolis on cryopreservation of common carp (Cyprinus carpio) sperm. Cryobiology 68: 107-112.

50. Öğretmen F, Inanan BE. 2014. Effect of butylated hydroxytoluene (BHT) on the cryopreservation of common carp (Cyprinus carpio) spermatozoa. Anim Reprod Sci 151: 269-274.

51. Öğretmen F, İnanan BE, Kutluyer F, Kayim M. 2015. Effect of semen extender supplementation with cysteine on postthaw sperm quality, DNA damage, and fertilizing ability in the common carp (Cyprinus carpio). Theriogenology 83: 1548-1552.

52. Osipova VP, Kolyada MN, Berberova NT, Milaeva ER, Ponomareva EN, Belaya MM. 2014. Cryoprotective effect of phosphorous-containing phenolic anti-oxidant for the cryopreservation of beluga sperm. Cryobiology 69: 467-472.

53. Rani PJ, Panneerselvam C. 2002. Effect of L-carnitine on brain lipid peroxidation and antioxidant enzymes in old rats. J Geront Series A (Biological and Medical Sciences) 57: 134-137.

54. Reddy NS, Mohanarao GJ, Atreja SK. 2010. Effects of adding taurine and trehalose to a tris-based egg yolk extender on buffalo (Bubalus bubalis) sperm quality following cryopreservation. Anim Reprod Sci 119: 183-190.

55. Robles VM, Santamaría YM, Casallas PE. 2005. Aspectos generales de la crioconservación espermática en peces teleósteos. Rev Col Cienc Pec 18: 34-48. 
56. Roca J, Gil MA, Hernandez M, Parrilla I, Vazquez JM, Martinez EA. 2004. Survival and fertility of boar spermatozoa after freeze-thawing in extender supplemented with butylated hydroxytoluene. J Androl 25: 397-405.

57. Roca J, Rodríguez MJ, Gil MA, Carvajal G, Garcia EM, Cuello C, Martinez E.A. 2005. Survival and in vitro fertility of boar spermatozoa frozen in the presence of superoxide dismutase and/or catalase. J Androl 26: 15-24.

58. Ruíz G, Santiani A, Sandoval M, Huanca L, Delgado C, Coronado S, Alzamora P. 2007. Efecto de dos antioxidantes (tempo y tempol) en la crioconservación de semen ovino empleando un dilutor en base a tris. Rev Investig Vet Perú 18: 99-106.

59. Russo A, Troncoso N, Sanchez F, Garbarino J, Vanella A. 2006. Propolis protects human spermatozoa from DNA damage caused by benzo [a] pyrene and exogenous reactive oxygen species. Life Sci 78: 1401-1406.

60. Saavedra OM, Vázquez EN, Vargas MR, Reyes GM, Bolaina EM. 2010. Radicales libres y su papel en las enfermedades crónico-degenerativas. Rev Méd Univ Veracruzana 10: 32-39.

61. Satorre MM, Breininger E, Beconi MT, Beorlegui NB. 2007. Tocopherol modifies tyrosine phosphorylation and capacitationlike state of cryopreserved porcine sperm. Theriogenology 68: 958-965.

62. Selley ML, Lacey MJ, Bartlett MR, Copeland CM, Ardlie NG. 1991. Content of significant amounts of a cytotoxic end-product of lipid peroxidation in human semen. $J$ Reprod \& Fertil 92: 291-298.

63. Sicherle CC, Maia MS, Bicudo SD, Rodello L, Azevedo HC. 2011. Lipid peroxidation and generation of hydrogen peroxide in frozen-thawed ram semen supplemented with catalase or trolox. Small Rum Res 95: 144-149.

64. Silva K, Moraes T, Silva E, Gamboa S, Guerra M. 2009. Efeito da adição de trolox e pentoxifilina na motilidade, integridade do acrossoma e do DNA de espermatozóides equinos após descongelação. Arq Bras Med Vet Zootec 61: 42-49.
65. Silva S, Soares A, Batista A, Almeida F, Nunes J, Peixoto C, Guerra M. 2011. In vitro and in vivo evaluation of ram sperm frozen in tris Egg-yolk and supplemented with superoxide dismutase and reduced glutathione. Reprod Dom Anim 46: 874-881.

66. Sobocanec S et al. 2006. Oxidant/antioxidant properties of croatian native propolis. J Agric \& Food Chem 54: 8018-8026.

67. Taylor K, Roberts P, Sanders K, Burton P. 2009. Effect of antioxidant supplementation of cryopreservation medium on post-thaw integrity of human spermatozoa. Reprod Biomed Online 18: 184-189.

68. Torres WH. 2002. Biología de las especies de oxígeno reactivas. Mens Bioquim 26: 19-53.

69. Tribble DL. 1999. Antioxidant consumption and risk of coronary heart disease. Circulation 99: 591-595.

70. Tuncer PB et al. 2010. The effect of raffinose and methionine on frozen/thawed Angora buck (Capra hircus ancryrensis) semen quality, lipid peroxidation and antioxidant enzyme activities. Cryobiology 61: 89-93.

71. Uysal O, Bucak M. 2007. Effects of oxidized glutathione, bovine serum albumin, cysteine and lycopene on the quality of frozen-thawed ram semen. Acta Vet Brno 76: 383-390.

72. Venereo JR. 2002. Daño oxidativo, radicales libres y antioxidantes. Rev Cubana Med Milit 31: 126-133.

73. Watson PF, Morris GJ. 1986. Cold shock injury in animal cells. Symp Soc Exp Biol 41: 311-340.

74. Yousef M, Abdallah G, Kamel K. 2003. Effect of ascorbic acid and vitamin $\mathrm{E}$ supplementation on semen quality and biochemical parameters of male rabbits. Anim Reprod Sci 76: 99-111.

75. Zilli L, Schiavone R, Zonno V, Rossano R, Storelli C, Vilella S. 2005. Effect of cryopreservation on sea bass sperm proteins. Biol Reprod 72: 1262-1267. 